12

\title{
Вращение плазменной струи высокочастотными электромагнитными полями и ее использование для масс-сепарации
}

\author{
(C) Н.М. Горшунов, ${ }^{1}$ Е.П. Потанин ${ }^{1,2}$ \\ ${ }^{1}$ Национальный исследовательский центр „Курчатовский институт“, \\ 123182 Москва, Россия \\ ${ }^{2}$ Национальный исследовательский ядерный университет „МИФИ“, \\ 115409 Москва, Россия \\ e-mail: potanin45@yandex.ru
}

Поступило в Редакцию 3 июля 2019 г.

В окончательной редакции 3 июля 2019 г.

Принято к публикации 16 сентября 2019 г.

Получены уравнения, описывающие характеристики азимутального движения и радиального расширения плазменной струи под действием вращающегося поперечного магнитного поля дипольной конфигурации в продольном статическом магнитном поле. Анализ проведен как в многокомпонентном приближении, так и на основе МГД-уравнений с учетом эффекта Холла. На основании полученных зависимостей окружной и радиальной скоростей ионов от величин магнитных полей проведены оценки разделительных характеристик прямоточной плазменной центрифуги при разделении двухкомпонентной бинарной смеси, моделирующей отработавшее ядерное топливо. Показано, что концентрация тяжелой уран-плутониевой компоненты в потоке отбора может быть увеличена от исходных 96 до 99.8\% при степени извлечения топливного компонента 0.87 .

Ключевые слова: плазма, вращение, электромагнитное поле.

DOI: $10.21883 /$ JTF.2020.03.48936.257-19

\section{Введение}

В настоящее время весьма актуальна проблема переработки отработавшего ядерного топлива (ОЯТ) атомных электростанций. Ее решение возможно на основе плазменных методов разделения ионов по массе [1-7]. Известно, что ОЯТ представляет собой весьма сложную смесь. Обычно в нее входит около 95\% урана и примерно $1 \%$ плутония - элементы с массой порядка 240 u. Остальное составляют радиоактивные продукты деления и прочие легкие элементы с атомными массами менее $160 \mathrm{u}$. Столь большое различие масс делает весьма заманчивым центробежное отделение легкой радиоактивной составляющей от ядерного топлива на одноступенчатой прямоточной установке, использующей радиальный разделительный эффект в быстро вращающейся плазменной струе, не контактирующей со стенкой. Один из возможных способов возбуждения центробежного поля сил в плазменном потоке заключается в создании конфигурации с взаимно перпендикулярными (скрещенными) постоянными электрическим и магнитным полями [3,8-12]. При таком способе неизбежно взаимодействие тока с электродами, что ведет к загрязнению плазмы и пристеночному торможению вращения. В работах [13-16] рассмотрен другой метод азимутального ускорения проводящего газа, основанный на использовании высокочастотной системы с вращающимся магнитным полем. Индукционный характер разряда позволяет в отличие от аналогичных устройств со скрещенными постоянными полями избежать применения электродных систем, а также снизить вероятность возникновения „спицевания“. Возможность возбуждения в проводящей среде азимутальных пондермоторных сил и связанной с ними раскрутки плазмы за счет действия ВЧ-полей при наличии осевого постоянного магнитного поля подтверждена экспериментально [15].

Целью настоящей работы является создание магнитогидродинамической модели стационарного вращения и расширения плазменной струи в осевом постоянном магнитном поле под действием вращающегося магнитного поля дипольной конфигурации. С использованием созданной расчетной модели проведены оценки газодинамических и разделительных характеристик вращающейся плазменной струи ионизированных продуктов ОЯТ.

\section{Описание принципиальной схемы процесса}

Упрощенная схема, обеспечивающая вращение и разделение компонентов плазменной струи по массам ионов, представлена на рис. 1. В отличие от работы [9], в которой исследуются характеристики противоточной циркуляционной плазменной центрифуги, в настоящей работе рассматривается прямоточный вариант разделительного устройства [17]. Предположим, что имеется источник сильно ионизованной плазмы продуктов ОЯТ 1 , прошедших первую стадию распада основных короткоживущих радионуклидов, создающий продольный плазменный поток. С точки зрения осуществления эффективного разделения и уменьшения потерь ядерного топлива на конструктивных элементах, необходимо стремиться 
к увеличению степени ионизации в зоне разделения. В плазме реального источника всегда будет присутствовать, пусть даже незначительная по концентрации, нейтральная компонента. Если источник плазмы вместе с системой разделения помещены в магнитное поле, то можно, располагая последнюю на некотором расстоянии от выхода источника, добиться увеличения степени ионизации плазмы в потоке, поскольку нейтральные частицы будут более эффективно диффундировать поперек магнитного поля по сравнению с заряженными частицами. Расположив на этом участке специальные карманы 2, в которые будут собираться нейтральные частицы, можно добиться повышения „качества“ разделяемой смеси. Предположим, что перед зоной разделения создан поток полностью ионизованной плазмы. Этот поток 3 поступает в зону разделения, в которой следует раскрутить плазму до скоростей, позволяющих эффективно отделить тяжелую составляющую смеси от легкой. Вращающееся поперечное магнитное поле увлекает плазму в азимутальном направлении за счет сфазированного взаимодействия индукционных осевого электрического тока и радиального магнитного поля. Следует ограничивать радиальное расширение плазменного столба 3, ведущее к взаимодействию плазмы со стенками камеры 4 и стержнями индукторов 6 .

Для замедления процесса ухода плазмы на стенки необходимо использовать достаточно высокую скорость осевого движения плазмы и достаточно сильное продольное магнитное поле В, создаваемое катушками 5. На конце рабочей зоны устанавливается специальный цилиндрический разделитель потока - скиммер 7, отделяющий поток тяжелых компонент смеси (уран и плутоний - тяжелая фракция 8) от легких (осколки деления и примеси - легкая фракция 9). Такую схему разделения принято называть прямоточной, в отличие от противоточной, обычно используемой в механических центрифугах [18].

Для создания вращающегося магнитного поля используется питаемая с соответствующим сдвигом фаз шестистержневая индукторная система 6 (рис. 1), создающая

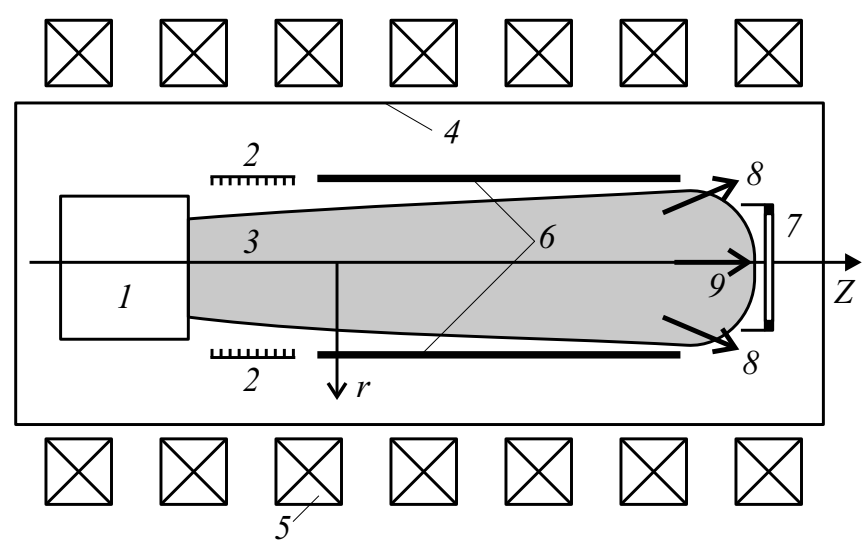

Рис. 1. Принципиальная схема устройства. Пояснения в тексте. в цилиндрическом объеме рабочей камеры магнитное поле дипольной конфигурации $\mathbf{B}_{0}$, направленное перпендикулярно оси и вращающееся с некоторой угловой скоростью $\omega[15,16]$. Вращающаяся компонента магнитного поля ускоряет плазму в азимутальном направлении. Ускорению плазмы препятствуют силы инерции. Радиальному разлету плазмы в основном препятствуют две силы: сила инерции, связанная с конвективным ускорением, и электромагнитная сила, обусловленная взаимодействием осевого магнитного поля с азимутальным током.

Нестационарность распределения плотности среды, вызванная радиальным расширением проводящего газа, существенно усложняет расчет. Однако если использовать достаточно сильное внешнее осевое магнитное поле, можно предположить, что радиальная скорость плазмы невелика, и в первом приближении не учитывать связанного с ней изменения во времени плотности среды, а также сил инерции в радиальном направлении, обусловленных конвективным ускорением.

\section{Анализ стационарного вращения и расширения плазменного столба}

Задача расчета гидродинамических параметров такого потока плазмы в достаточно общей постановке состоит в решении системы нелинейных дифференциальных уравнений в частных производных с учетом нестационарного процесса ускорения среды в условиях одновременного действия электромагнитных сил, связанных как с внешним осевым удерживающим магнитным полем, так и с ВЧ-ускоряющим полем. Заменим реальное дискретное распределение осевых токов в стержневой системе непрерывным бегущим токовым слоем [16]. Предположим, что линейная плотность поверхностного тока в слое изменяется по гармоническому закону

$$
\tilde{J}_{z}=J_{z 0} \exp [i(\omega t-\varphi)]
$$

где $J_{z 0}$ - линейная плотность поверхностного тока, $\omega$ - круговая частота.

Такое распределение соответствует вращающемуся с постоянной угловой скоростью поперечному оси однородному магнитному полю дипольной конфигурации, раскручивающему плазменный столб за счет электромагнитных сил. Пусть на плазму, помимо поперечных переменных магнитных полей $\tilde{B}_{r}$ и $\tilde{B}_{\varphi}$, связанных с плотностью переменного тока $\tilde{J}_{z}$, наложено постоянное осевое магнитное поле $B_{z}$, создаваемое катушками 5 (рис. 1). Поток плазмы после прохождения зоны предварительного расширения 2 (рис. 1) направляется в основную рабочую область (зона разделения), в которой действуют продольное однородное магнитное поле $B_{z}$ и перпендикулярное к нему ВЧ-вращающееся магнитное поле $\left(\tilde{B}_{r}, \tilde{B}_{\varphi}\right)$ дипольной конфигурации. 
Пусть направления внешнего магнитного поля $\mathbf{B}$ и угловой скорости вращения поперечного поля $\boldsymbol{\omega}$ совпадают $\boldsymbol{\omega} \uparrow \uparrow \mathbf{B})$. Величины переменных электромагнитных полей, связанных с действием вращающегося магнитного поля и слабо искажающихся за счет токов в плазме (эффект скинирования), равны [19]:

$$
\begin{gathered}
\tilde{B}_{r}=B_{0} \cos (\omega t-\varphi), \quad \tilde{B}_{\varphi}=B_{0} \sin (\omega t-\varphi), \\
\tilde{E}_{z}=B_{0} \omega r \cos (\omega t-\varphi) .
\end{gathered}
$$

Будем предполагать вращение устойчивым и пренебрегать какими-либо явлениями, связанными с взаимодействием плазмы с торцевыми тормозящими элементами конструкции [20,21]. Выполним сначала расчет компонент скоростей вращения и радиального расширения ионной и электронной компонент полностью ионизованной плазмы в рамках многокомпонентного гидродинамического приближения. В рассматриваемом случае уравнения сохранения импульса для ионов и электронов должны учитывать кроме сил Лоренца также члены, обусловленные взаимным трением между компонентами, и нелинейные инерционные члены, связанные с расширением плазменного столба:

$$
\begin{aligned}
m_{i} n_{i} \frac{d \mathbf{V}_{i}}{d t}= & -\operatorname{grad}\left(p_{i}\right)+n_{i} e\left(\mathbf{E}+\mathbf{V}_{i} \times \mathbf{B}\right) \\
& -n^{2} \alpha\left(\mathbf{V}_{i}-\mathbf{V}_{e}\right) \\
m_{e} n_{e} \frac{d \mathbf{V}_{e}}{d t}= & -\operatorname{grad}\left(p_{e}\right)-n_{e} e\left(\mathbf{E}+\mathbf{V}_{e} \times \mathbf{B}\right) \\
& -n^{2} \alpha\left(\mathbf{V}_{i}-\mathbf{V}_{e}\right),
\end{aligned}
$$

где $n=n_{e} \cong n_{i}-$ плотность частиц; $\alpha-$ коэффициент, характеризующий силы диффузионного трения между ионами и электронами. Знак $\frac{d}{d t}$ означает полную производную. Обозначим $u_{k}, v_{k}$ и $w_{k}$ радиальную, азимутальную и осевую компоненты скоростей ионов $(k=i)$ и электронов $(k=e)$. Представим эти компоненты и величину $E_{r}$ в виде сумм, медленно изменяющихся во времени, и быстро осциллирующих составляющих $u_{k}=\left\langle u_{k}\right\rangle+\tilde{u}_{k}, v_{k}=\left\langle v_{k}\right\rangle+\tilde{v}_{k}$, $w_{k}=\left\langle w_{k}\right\rangle+\tilde{w}_{k}, \quad E_{r}=\left\langle E_{r}\right\rangle+\tilde{E}_{r}$. Положим $\tilde{u}_{k} \ll\langle u\rangle_{k}$, $\tilde{v}_{k} \ll\left\langle v_{k}\right\rangle, \tilde{E}_{r} \ll\left\langle E_{r}\right\rangle,\left\langle w_{k}\right\rangle=0$. Поскольку в достаточно сильном продольном магнитном поле $B_{z} \gg B_{0}$ радиальное расширение столба затруднено, в радиальной проекции уравнения (3) пренебрегаем составляющими инерционных ускорений ионов, квадратичных по $\left\langle u_{i}\right\rangle$, оставив только центробежный член. Рассмотрим стационарный режим для радиального и азимутального движений, учтем малость инерционных ускорений электронов и индуцированных магнитных полей. Кроме того, в силу свободного характера расширения плазменного столба и отсутствия его взаимодействия со стенками устройства, пренебрежем градиентами давления заряженных частиц. С учетом условия $u_{e}=u_{i}$ (внешняя электрическая цепь разомкнута, вследствие чего радиальный электрический ток отсутствует) на основании (4) в проекции на радиальное направление имеем

$$
\left\langle v_{e}\right\rangle=-\frac{\left\langle E_{r}\right\rangle}{B_{z}} .
$$

Используя выражение для $\left\langle E_{r}\right\rangle$ из (5) и учитывая, что $\tilde{E}_{z}=\omega r \tilde{B}_{r}$, а также производя усреднение электромагнитных сил, действующих на ионы и электроны по периоду колебаний ВЧ-поля, получим

$$
\begin{gathered}
-m_{i} \frac{\left\langle v_{i}\right\rangle^{2}}{r}=e B_{z}\left(\left\langle v_{i}\right\rangle-\left\langle v_{e}\right\rangle\right), \\
m_{i} n\left\langle u_{i}\right\rangle \frac{\partial\left\langle v_{i}\right\rangle}{\partial r}+m_{i} \frac{\left\langle v_{i}\right\rangle\left\langle u_{i}\right\rangle}{r}=-e\left\langle u_{i}\right\rangle B_{z}+e\left\langle\tilde{w}_{i} \tilde{B}_{r}\right\rangle \\
-n \alpha\left(\left\langle v_{i}\right\rangle-\left\langle v_{e}\right\rangle\right), \\
m_{i} \frac{\partial \tilde{w}_{i}}{\partial t}+m_{i} \frac{v_{i}}{r} \frac{\partial \tilde{w}_{i}}{\partial \varphi}=e \tilde{B}_{r}\left(\omega r-\left\langle v_{i}\right\rangle\right)-n \alpha\left(\tilde{w}_{i}-\tilde{w}_{e}\right), \\
0=e\left\langle u_{i}\right\rangle B_{z}-e\left\langle\tilde{w}_{e} \tilde{B}_{r}\right\rangle-n \alpha\left(\left\langle v_{e}\right\rangle-\left\langle v_{i}\right\rangle\right), \\
-m_{e} \frac{\partial \tilde{w}_{e}}{\partial t}+m_{i} \frac{v_{i}}{r} \frac{\partial \tilde{w}_{e}}{\partial \varphi}=e \tilde{B}_{r}\left(\omega r-\left\langle v_{e}\right\rangle\right)+n \alpha\left(\tilde{w}_{e}-\tilde{w}_{i}\right) .
\end{gathered}
$$

Будем искать решение системы уравнений (6)-(10) в виде

$$
\begin{gathered}
\left\langle v_{i}\right\rangle=r \Omega_{i}, \quad\left\langle v_{e}\right\rangle=r \Omega_{e}, \quad\left\langle u_{i}\right\rangle=r U_{i}, \\
\tilde{w}_{i}=r \tilde{W}_{i}, \quad \tilde{w}_{e}=r \tilde{W}_{e} .
\end{gathered}
$$

Решение системы уравнений (8) и (10) с учетом (2) дает

$$
\tilde{W}_{e}=A_{1} \sin \omega t+C_{1} \cos \omega t, \quad \tilde{W}_{i}=A_{2} \sin \omega t+C_{2} \cos \omega t,
$$

где

$$
\begin{aligned}
A_{1}= & -\tau_{1}(\omega-\Omega) \\
& +\frac{\left(R M+M^{2}\right)\left[t_{1}\left(\omega-\Omega_{e}\right)+t_{2}\left(\omega-\Omega_{i}\right)\right]}{\left(M^{2}+2 R M+R^{2}+1\right)}, \\
A_{2}=- & \tau_{1}(\omega-\Omega) \\
+ & \frac{\left(R M+M^{2}+1\right)\left[t_{1}\left(\omega-\Omega_{e}\right)+t_{2}\left(\omega-\Omega_{i}\right)\right]}{\left(M^{2}+2 R M+E^{2}+1\right)}, \\
& C_{1}=\frac{M\left[t_{1}\left(\omega-\Omega_{e}\right)+t_{2}\left(\omega-\Omega_{i}\right)\right]}{\left(M^{2}+2 R M+R^{2}+1\right)}, \\
C_{2}=\frac{R\left[t_{1}\left(\omega-\Omega_{e}\right)+t_{2}\left(\omega-\Omega_{i}\right)\right]}{\left(M^{2}+2 R M+R^{2}+1\right)}, & \frac{e B_{0}}{m_{e} \omega}, \quad M=\frac{n \alpha}{m_{e} \omega}, \quad R=\frac{n \alpha}{m_{i} \omega} .
\end{aligned}
$$

Используя (6), (7), (9), (11) вместе с неравенством $\frac{m_{e}}{m_{i}} \ll 1, \Omega_{e} \ll \omega$ и предположением о малости угловой скорости $\omega$ и ионной циклотронной частоты $\omega_{i c}=\frac{e B_{z}}{m_{i}}$ 
по сравнению с частотой электрон-ионных соударений $v_{e i}$, вводя безразмерные величины $y=\frac{\Omega_{i}}{\omega}, x=\frac{U_{i}}{\omega}$, $S=\frac{e^{2} B_{0}^{2}}{2 m_{i} n \alpha \omega}=\frac{\sigma_{0} B_{0}^{2}}{2 \rho_{i} \omega}$, а также массовую плотность ионной компоненты $\rho_{i}$ и проводимость плазмы вдоль магнитного поля $\sigma_{0}$, получим

$$
\begin{gathered}
2 x y=S \frac{M^{2}}{M^{2}+1}\left(1-y-y^{2} \frac{\omega}{\omega_{c}}\right), \\
x\left(1+2 y \frac{\omega}{\omega_{c}}\right)=d \frac{y^{2}}{S} .
\end{gathered}
$$

Уравнения (12), (13) описывают гидродинамические характеристики ионного компонента плазмы под действием вращающегося магнитного поля в сильном продольном статическом магнитном поле в многокомпонентном гидродинамическом приближении.

Прежде чем переходить к непосредственному решению уравнений (12), (13), рассмотрим эту задачу в более простом, однокомпонентном гидродинамическом приближении, учитывая тем не менее холловские токи. Систему МГД-уравнений движения полностью ионизованной плазмы совместно с обобщенным законом Ома запишем в виде [22,23]

$$
\begin{gathered}
(\mathbf{V} \nabla) \mathbf{V}=-\frac{1}{\rho} \nabla p+\frac{1}{\rho} \mathbf{j} \times \mathbf{B}, \\
\frac{1}{v_{e i}} \frac{\partial \mathbf{j}}{\partial t}+\mathbf{j}=\sigma_{0}[\mathbf{E}+\mathbf{V} \times \mathbf{B}-\beta \mathbf{j} \times \mathbf{B}],
\end{gathered}
$$

где $\mathbf{V}$ - среднемассовая скорость плазмы, $p$ - давление, $\mathbf{j}$ - плотность электрического тока, В - магнитная индукция, $\mathbf{E}-$ напряженность электрического поля, $\beta$ - постоянная Холла, $\rho-$ массовая плотность среды.

Система уравнений (14)-(15) при сделанных выше предположениях в проекциях на оси $r$ и $\varphi$ запишется в виде

$$
\begin{gathered}
-\rho \frac{V_{\varphi}^{2}}{r}=j_{\varphi} B_{z}-j_{z} B_{\varphi}, \\
\rho V_{r} \frac{\partial V_{r}}{\partial r}+\rho \frac{V_{r} V_{\varphi}}{r}=j_{z} B_{r} .
\end{gathered}
$$

Ускорение проводящей среды вращающимся магнитным полем связано с возбуждением в плазме объемной электромагнитной силы $F_{\varphi}=j_{z} B_{r}$, обусловленной взаимодействием осевых токов с радиальной компонентой магнитного поля. Представляя величины плотности азимутального электрического тока $j_{\varphi}$, а также азимутальной $v$ и радиальной $u$ скоростей плазмы в виде суммы медленно изменяющихся во времени и быстро осциллирующих составляющих и проводя усреднение с учетом малости последних, приходим к

$$
\begin{gathered}
\tilde{j}_{z}=\frac{\sigma_{0} \omega r B_{0}}{C_{3}} A_{3} \cos (\omega t-\varphi)+\frac{\sigma_{0} \omega^{2} r B_{0}}{\nu_{e i} C_{3}} A_{3} \sin (\omega t-\varphi), \\
\left\langle j_{\varphi}\right\rangle=\sigma_{0}\left(\langle u\rangle B_{z}-\frac{\sigma_{0} \beta r B_{0}^{2} A_{3}}{2 C_{3}}\right)
\end{gathered}
$$

где $\quad C_{3}=1+\omega^{2} / \nu_{e i}^{2}+\sigma_{0}^{2} \beta^{2} B_{0}^{2} / 2, \quad A_{3}=1-\langle v\rangle / \omega r-$ $-\sigma_{0} \beta B_{z} / \omega r$. Используя (16), (17), а также (18), (19), найдем

$$
\begin{aligned}
2 x y\left(1+N+\chi^{2}\right) & =S\left(1-y-\chi x / d^{1 / 2}\right), \\
y^{2}\left(1+N+\chi^{2}\right) d & =S\left(x+\chi(1-y) d^{1 / 2}\right),
\end{aligned}
$$

где $y=\langle v\rangle / \omega r, \quad x=\langle u\rangle / \omega r, \quad S=\sigma_{0} B_{0}^{2} / 2 \rho \omega, \quad N=\frac{\omega^{2}}{v_{e i}^{2}}$, $d=B_{0}^{2} / 2 B_{z}^{2}, \chi=\sigma_{0} \beta B_{0} / \sqrt{2}$.

Сравним системы уравнений (12), (13) и (20), (21). Простые алгебраические действия показывают, что при $N=\omega^{2} / \nu_{e i}^{2} \ll 1,\left(M^{2}=1 / N \gg 1\right)$ они полностью эквивалентны. Таким образом, применительно к рассматриваемой задаче магнитогидродинамическое приближение с учетом эффекта Холла в достаточно плотной плазме соответствует многокомпонентному гидродинамическому приближению, если под гидродинамической среднемассовой скоростью плазмы понимать скорость ионов. Однако многокомпонентное приближение позволяет дополнительно определить и скорости электронной компоненты плазмы.

Из (20) и (21) имеем уравнение для определения $y$

$$
\begin{aligned}
& \frac{2 d\left(1+N+\chi^{2}\right)^{2} y^{3}}{S^{2}\left(1+\chi^{2}+2\left(1+N+\chi^{2}\right) y \omega / \omega_{c}\right)}= \\
& =\left(1+\chi^{2}\right)(1-y)-\left(1+N+\chi^{2}\right) y^{2} \omega / \omega_{c} .
\end{aligned}
$$

\section{Расчет гидродинамических параметров плазмы применительно к разделению ОЯТ}

В качестве модельной смеси рассмотрим бинарную смесь с атомными весами 120 и $240 \mathrm{u}$. Преобладающим компонентом смеси будем считать атомы с массовым числом, близким к 240, в результате чего средняя молярная масса смеси составляет $\langle\mu\rangle \sim 240 \mathrm{~kg} / \mathrm{kmol}$. Положим проводимость плазмы вдоль магнитного поля $\sigma_{0}=10^{3} 1 /(\Omega \cdot \mathrm{m})$ (проводимость полностью ионизованной плазмы при температуре электронов $T_{e} \sim 1 \mathrm{eV}$ ), угловую скорость вращения поперечного магнитного поля $\omega=10^{6} \mathrm{~s}^{-1}$, радиус столба $R=5 \cdot 10^{-2} \mathrm{~m}$.

Результаты решения уравнения (22), а также последующего нахождения величины безразмерной радиальной и азимутальной скоростей представлены на рис. 2 и 3. C ростом постоянного магнитного поля $B_{z}$ скорость радиального расширения уменьшается. На рис. 2 приведены результаты расчета зависимости безразмерной радиальной скорости плазмы $x$ в зависимости от величины $B_{z}$ для плотности плазмы $n=10^{19} 1 / \mathrm{m}^{3}$ и амплитуды высокочастотного поля $B_{0}=10^{-3}$ Т. На рис. 3. показаны зависимости безразмерной азимутальной скорости $y$ от величины $B_{z}$ для различных значений $B_{0}$ при плотности плазмы $n=10^{18} 1 / \mathrm{m}^{3}$. Снижение радиальной скорости расширения с возрастанием величины $B_{0}$ связано с 


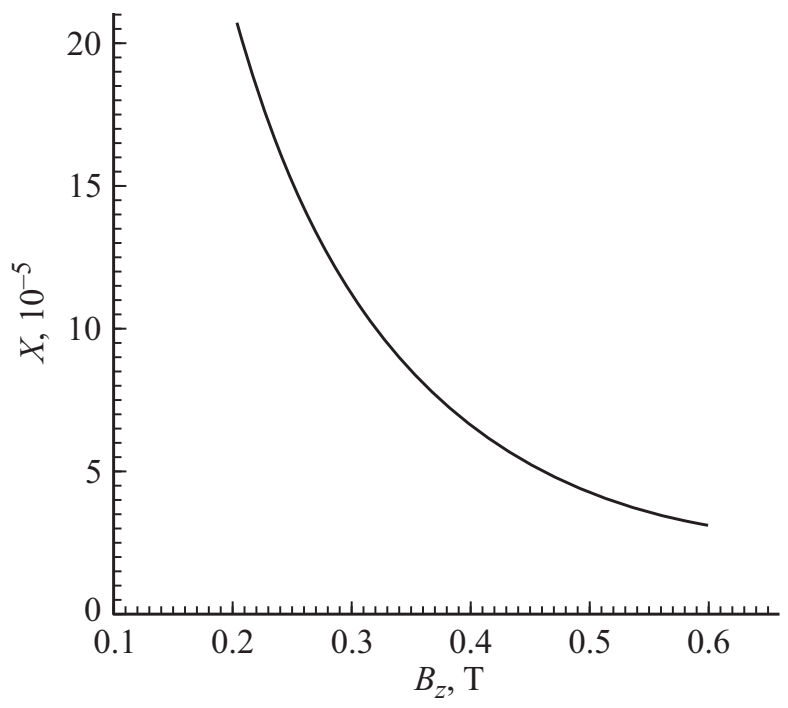

Рис. 2. Зависимость безразмерной радиальной скорости плазменного столба $x$ от индукции продольного статического магнитного поля $B_{z}$ для $n=10^{19} 1 / \mathrm{m}^{3}$ и $r=0.05 \mathrm{~m}$.

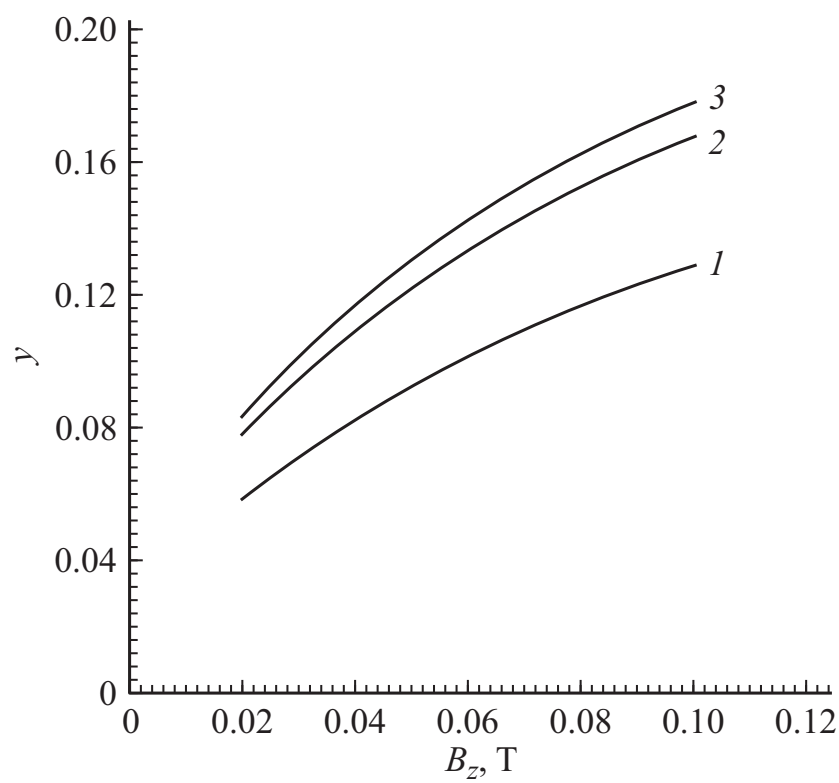

Рис. 3. Зависимость безразмерной азимутальной скорости $y$ плазменного столба от индукции продольного статического магнитного поля $B_{z}$ для $n=10^{18} 1 / \mathrm{m}^{3}, r=0.05 \mathrm{~m}$ при различных значениях амплитуды переменного магнитного поля $B_{0}$ : $1-2 \cdot 10^{-4}, 2-5 \cdot 10^{-4}$, и $3-10^{-3} \mathrm{~T}$.

преобладающим влиянием при рассматриваемых параметрах плазмы азимутального холловского тока и его взаимодействием с продольным магнитным полем $B_{z}$.

Оценим справедливость пренебрежения эффектами скинирования высокочастотного поля. Толщина скинслоя может быть оценена в соответствии с выражением $\delta \sim \sqrt{2\left(1+\chi^{2}\right) / \sigma_{0} \mu_{0} \omega}$. Полагая $\sigma_{0}=10^{3} 1 /(\Omega \cdot \mathrm{m})$, $\omega=10^{6} 1 / \mathrm{s}, \quad \chi=4.4$, получим $\delta \sim 0.2 \mathrm{~m}$, что существенно превышает величину $R_{0}$. Время установления окружной скорости плазмы $\tau_{\varphi}$ может быть оценено из баланса инерционных членов и электромагнитных сил как $\tau_{\varphi} \sim \rho\left(1+\chi^{2}\right) y / \sigma_{0} B_{0}^{2}$. Полагая для режима c $\rho=4 \cdot 10^{-7} \mathrm{~kg} / \mathrm{m}^{3}, B_{0}=10^{-3} \mathrm{~T}, B_{z}=0.05 \mathrm{~T}, \chi=4.4$, $y=0.013$, получим $\tau_{\varphi} \sim 10^{-3} \mathrm{~s}$.

Оценим время диффузионого процесса $t_{d}$. Для цилиндрической геометрии имеем $\tau_{d} \sim \frac{R_{0}^{2}}{5 D_{i i}}$, где $D_{i i}-$ коэффициент радиальной взаимной диффузии изотопных ионов. Используем для средней частоты столкновений между ионами соотношение $v_{i i}=\lambda e^{4} n / 12 \sqrt{m_{i}} \times$ $\times\left(k_{i}\left\langle T_{i}\right\rangle\right)^{3 / 2} \pi^{3 / 2} \varepsilon_{0}^{2}$ [24], где $T-$ температура ионов, $\lambda$ - кулоновский логарифм, $\varepsilon_{0}$ - электрическая постоянная. Коэффициент радиальной диффузии оценим как $D_{i i} \sim k T_{i} / m_{i} v_{i i} . \quad$ Полагая $\left\langle T_{i}\right\rangle=10^{4} \mathrm{~K}, \quad \lambda=10, \quad n=$ $=10^{18} 1 / \mathrm{m}^{3}$, получим $\tau_{d} \sim 10^{-4} \mathrm{~s}$. Скорость продольного плазменного потока положим равной скорости ионного звука $V_{z}=\sqrt{k T_{e} / m}$. При длине зоны разделения $L=1 \mathrm{~m}$ найдем для времени пролета $\tau_{f}=L / V_{z} \sim 2 \cdot 10^{-3} \mathrm{~s}$, что превышает оценки для $\tau_{d}=10^{-4} \mathrm{~s}$ и $\tau_{\varphi}=10^{-3} \mathrm{~s}$. Радиальная скорость расширения плазменного столба при $n=10^{18} 1 / \mathrm{m}^{3}, B_{z}=0.1 \mathrm{~T}$ и $B_{0}=2 \cdot 10^{-4} \mathrm{~T}$ составляет около $2 \mathrm{~m} / \mathrm{s}$. Следовательно, расширение плазменного столба за время пролета плазмой зоны разделения не превышает $\Delta r=4 \mathrm{~mm}$. Поэтому уходом частиц плазмы на боковые стенки и поверхность стержней индуктора будем пренебрегать.

\section{Оценка разделительных характеристик прямоточной плазменной центрифуги}

Для выяснения возможности отделения тяжелых ионов со средним массовым числом $240 \mathrm{u}$ от легких ионов с массовым числом $120 \mathrm{u}$ на индукционной центрифуге проведем оценку степени чистоты топливных элементов в потоке отбора рассматриваемой установки (рис. 1). Отметим, что, как показывают расчеты, в

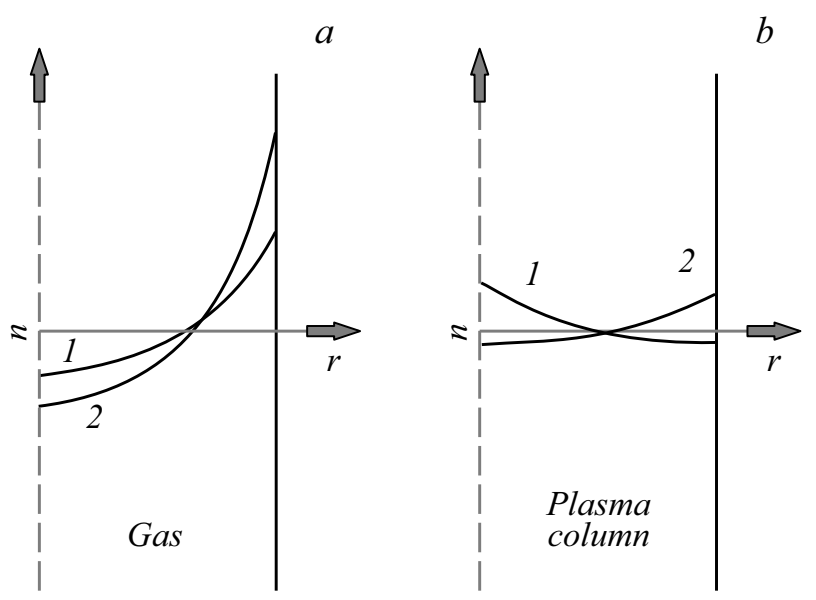

Рис. 4. Качественные профили парциальной плотности компонентов бинарной смеси в механической $(a)$ и плазменной $(b)$ прямоточных центрифугах: 1 и 2 - плотности легкой и тяжелой компонент. 
достаточно сильном продольном магнитном поле центробежная сила в прямоточной системе может быть уравновешена электромагнитной. Ситуация здесь существенно отличается от случая механической центрифуги, в которой центробежная сила уравновешивается силой, связанной с градиентом давления. На рис. 4 приведены качественные радиальные профили парциальных плотностей компонентов бинарной смеси в механической и плазменной центрифугах. Разделение компонент смеси по радиусу происходит в условиях противодействия массовых центробежных сил и не зависящих от массы электромагнитных сил. В результате распределение концентрации по радиусу описывается зависимостью

$$
\frac{(c /(1-c))_{r}}{(c /(1-c))_{r=0}}=\exp \left\{\int_{0}^{r} \Delta m\langle v\rangle^{2} r k T d r\right\},
$$

где $c$ - мольная концентрация ионов тяжелого компонента смеси на радиусе $r, \Delta m-$ разность масс ионов разделяемых компонентов, $k$ - постоянная Больцмана.

Интегрируя (23) с учетом $\left\langle v_{i}\right\rangle=r \Omega_{i}$ и решая полученное уравнение относительно $c$, получаем зависимость концентрации топливной компоненты от радиуса

$$
c=a \exp \left(b r^{2}\right) /\left[1+a \exp \left(b r^{2}\right)\right]
$$

где $a=c_{0} /\left(1-c_{0}\right), b=\Delta m \Omega_{i}^{2} / 2 k T, c_{0}-$ концентрация на оси.

Радиус скиммера $r_{S}$ выбираем так, чтобы при $r=r_{S}$ концентрация тяжелого компонента $c_{s}$ равнялась исходной. Тогда из (24) получаем $r_{s}^{2}=1 / b \ln \left[c_{s} / a\left(1-c_{s}\right)\right]$.

Полагая $n=10^{18} 1 / \mathrm{m}^{3}, \quad B_{0}=2 \cdot 10^{-4} \mathrm{~T}, \quad B_{z}=0.1 \mathrm{~T}$, $T_{i}=10^{4} \mathrm{~K}, \quad c_{s}=0.96, \Delta m=2 \cdot 10^{-25} \mathrm{~kg} \quad$ и используя оцененные выше значения скорости вращения плазмы $\left(\Omega_{i} \approx 1.3 \cdot 10^{5} 1 / \mathrm{s}\right)$ и условие сохранения полного количества тяжелых ионов на единицу длины струи, найдем численное значение концентрации обогащенной топливной компоненты в потоке отбора $\left(r \geq r_{s}\right)$ $c^{+}=0.998$, что соответствует уменьшению в отборе легкой радиоактивной компоненты в 20 раз. Степень извлечения топливного компонента, рассчитываемая как $\Gamma=c^{+}\left(R_{0}^{2}-r_{s}^{2}\right) / c_{s} R_{0}^{2}$, равна 0.87. С возрастанием радиуса скиммера концентрация обогащенной топливной компоненты будет расти, а степень извлечения уменьшаться.

\section{Заключение}

Предлагается магнитогидродинамическая модель расчета стационарного вращения и расширения плазменной струи в осевом постоянном магнитном поле под действием индукционного вращающегося магнитного поля дипольной конфигурации. С использованием созданной расчетной модели проведены оценки характеристик разделения полностью ионизированного потока ОЯТ во вращающейся плазменной струе, не взаимодействующей со стенками камеры. Такая конструкция соответствует прямоточной центрифуге. Показано, что при вырезании из вращающегося плазменного цилиндра диаметром $10 \mathrm{~cm}$ центральной части с помощью кольцевого скиммера диаметром $2 \mathrm{~cm}$ концентрация обогащенной топливной компоненты в потоке отбора возрастает от исходной 0.96 до 0.998. При этом степень извлечения топливных компонент составляет 0.87 .

Полученные результаты указывают на целесообразность дальнейшего исследования возможности переработки ОЯТ на индукционных одноступенчатых прямоточных плазменных центрифугах.

\section{Благодарности}

Авторы признательны Пустовитову В.Д. и Тимофееву А.В. за полезные замечания.

\section{Финансирование работы}

Е.П. Потанин благодарен за частичную финансовую поддержку Программы повышения конкурентноспособности Национальный исследовательский ядерный университет МИФИ (контракт № 02.a03.21.0005 от 27.08.2013).

\section{Конфликт интересов}

Авторы заявляют, что у них нет конфликта интересов.

\section{Список литературы}

[1] Смирнов В.П., Жильцов В.А., Морозов А.И., Семашко Н.Н., Сковорода А.А., Тимофеев А.В. // Сб. докладов на IX Всероссийской (международной) научной конф. „Физико-химические процессы при селекции атомов и молекул“ 4-8 октября 2004. Звенигород. С. 7-11.

[2] Жильцов В.А., Кульгин В.М., Семашко Н.Н., Сковорода А.А., Смирнов В.П., Тимофеев А.В., Кудрявцев Е.Г., Рачков В.И., Орлов В.В. // Атомная энергия. 2006. Т. 101. Вып. 4. C. 302-306.

[3] Rax J.-M., Gueroult R. // J. Plasma Phys. 2016. Vol. 82. P. 595820504.

[4] Тимофеев А.В. // УФН. 2014. Т. 184. № 10. С. 1101-1133.

[5] Самохин А.А., Смирнов В.П., Гавриков А.В., Ворона Н.А. // ЖТФ. 2016. Т. 86. Вып. 2. С. 127-132.

[6] Амиров Р.Х., Ворона Н.А., Гавриков А.В., Жабин С.Н., Лизякин Г.Д., Полищук В.П., Самойлов И.С., Смирнов В.П., Усманов Р.А., Ярцев И.М. // Труды МФТИ. 2014. Т. 6. № 1. C. $136-145$.

[7] Усманов Р.А., Лизякин Г.Д., Полищук В.П., Яриев И.М. // Физическое образование в вузах. 2016. Т. 22. № 1. C. $142-143$

[8] Карчевский А.И., Потанин Е.П. Плазменные центрифуги. В кн. Изотопы. / Под. ред. В.Ю. Баранова. М.: Изд-во АТ, 2005. T. 1. С. $326-338$.

[9] Борисевич В.Д., Потанин Е.П. // ЖТФ. 2018. Т. 88. Вып. 5. С. 791-794.

[10] James B.W., Simpson S.W. // Plasma Phys. 1976. Vol. 18. P. 289-300. 
[11] Белорусов А.В., Карчевский А.И., Муромкин Ю.А., Потанин Е.П. // Письма в ЖТФ. 1976. Т. 2. Вып. 14. С. 664-668.

[12] Fetterman A.J., Fisch N.J. // Phys. Plasmas. 2011. P. 094503.

[13] Аверин В.Г., Белорусов А.В., Карчевский А.И., Марцынкьян В.ЛІ., Попов И.А., Потанин Е.П. // ЖТФ. 1978. Т. 48. Вып. 1. C. 66-71.

[14] Borisevich V.D., Potanin E.P. // Phys. Scripta. 2017. Vol. 92. P. 075601.

[15] Жариков В.Н., Миненко В.П. // Физика плазмы. 1988. T. 14. № 7. C. $1122-1126$.

[16] Горшунов Н.М., Потанин Е.П. // Атомная энергия. 2018. Т. 124. Вып. 8. С. 164-169.

[17] Токманщев В.И., Палкин В.А. // Атомная энергия. 2017. T. 123. Вып. 1. С. 40-45

[18] Борисевич В.Д., Борман В.Д., Сулаберидзе Г.А., Тихомиров А.В., Токманщев В.И. // Физические основы разделения изотопов в газоцентрифуге. М.: Издат. дом МЭИ, 2011. $275 \mathrm{c}$.

[19] Карчевский А.И., Потанин Е.П. // Магнитная гидродинамика. 1991. № 3. С. 103.

[20] Горбачев Л.П., Никитин Н.В., Устинов А.Р. // Магнитная гидродинамика. 1974. № 4. C. 32-43.

[21] Borisevich V.D., Potanin E.P., Whichello J. // J. Fluid Mech. 2017. Vol. 829. P. 328-244.

[22] Франк-Каменецкий Д.А. Лекции по физике плазмы. М.: Атомиздат, 1968. $286 \mathrm{c.}$

[23] Саттон Джс., Шерман А. Основы технической магнитной газодинамики. М.: Мир, 1968. 492 с.

[24] Брагинский С.И. Вопросы теории плазмы / Под. ред. М.А. Леонтовича. М.: Госатомиздат, 1963. Вып. 1. С. 183. 